\title{
IMPLEMENTACIÓN DE ENSANCHADORES Y CENTRALIZADORES PROTECH CRB PARA MEJORAR VIAJES Y CORRIDAS DE REVESTIMIENTO EN POZOS DEL PIEDEMONTE LLANERO COLOMBIANO
}

Zoraya Ramírez Sepúlveda ${ }^{1}$, Sergio Alberto López Anaya² *, Iván Fernando Palacios Mendoza ${ }^{3}$

*A quien va dirigida la correspondencia

\section{RESUMEN}

El objetivo de este trabajo es evaluar técnicamente dos nuevas tecnologías (ensanchadores y centralizadores Protech $C R B$ ) implementadas por la compañía Equión Energía Limitada para reducir tiempos de viajes y tiempos de corridas de revestimientos durante la perforación de pozos en el Piedemonte Llanero Colombiano.

Para lograr lo anterior, se reunió y limpió la información necesaria de los reportes diarios de perforación de ocho pozos de correlación (offset) seleccionados y se comparó con la de los dos pozos donde se implementaron las tecnologías. Datos de tiempos y velocidades de viajes y corridas de revestimientos, tasas de penetración (ROP) y tiempos planos (flat times) fueron analizados y posteriormente cuantificados, para determinar el impacto operacional y económico de los ensanchamientos de hueco y las corridas con centralizadores Protech CRB.

En los resultados obtenidos, se observan los beneficios alcanzados con la implementación de las nuevas tecnologías y su rentabilidad. Se destaca su aporte a las buenas condiciones del pozo, que reducen riesgos comunes de presentar tiempos no productivos (NPT) durante las actividades de perforación, relacionados con eventos de pega de tubería, pérdida de integridad del revestimiento y trabajos remediales de cementación, y los costos adicionales que pueden generar.

Palabras clave: Tiempos de viaje, Corridas de revestimiento, Ensanchador, Centralizadores Protech CRB, Puntos apretados.

\section{IMPLEMENTATION OF UNDERREAMERS AND PROTECH CRB CENTRALIZERS TO IMPROVE TRIPPING AND CASING RUN OPERATIONS AT COLOMBIAN PIEDEMONTE LLANERO OILFIELDS}

\footnotetext{
ABSTRACT

The aim of this project is the technical evaluation of two new technologies (hole enlargers and Protech CRB centralizers) implemented by the company Equion Energia Limited to reduce pipe tripping and casing/liner running times during the drilling of wells at Colombian Piedemonte Llanero oilfields.

In order to accomplish this goal, information contained in daily drilling reports from eight different offset wells was gathered and compared to the one from the two wells were technologies were applied. Data of time summaries, pipe tripping and casing/liner run velocities, as well as rates of penetration (ROP) and flat times were analyzed

1. Ingeniera de Petróleos. Líder de Nuevas Tecnologías de Perforación y Completamiento, Equión Energía Ltda. E-mail: Zoraya.Ramirez@equion-energia.com

2. Ingeniero de Petróleos, Universidad Industrial de Santander. Bucaramanga, Colombia. E-mail: sergiolopez9012@ gmail.com

3. Ingeniero de Petróleos, Universidad Industrial de Santander. Bucaramanga, Colombia. E-mail: ivanfernandopm@ yahoo.com
} 
and therefore quantified to determine the operational and economic impact of hole enlargements and Protech CRB centralizers performance.

Finally, this project results clearly show the advantages reached with the implementation of the new technologies and its profit. It is important to highlight their contribution to improve hole conditions, which reduce common risks that could lead to have non-productive times (NPT) during drilling operations. For example, stuck pipe events, casing integrity failure and remedial cementing jobs, along with the additional costs they may generate.

Keywords: Trip times, Casing/liner run, Underreamer, Protech CRB centralizers, tight spots.

\section{INTRODUCCIÓN}

Previo a la evaluación técnica de la implementación de nuevas tecnologías en la perforación de pozos petrolíferos, existe una etapa preliminar que corresponde al análisis de pozos de correlación, en la cual se estudian pozos análogos respecto a pozos de interés para determinar los problemas operacionales que se presentaron durante la perforación, las soluciones ejecutadas y los resultados obtenidos. Este proceso es vital en la elaboración de todo proyecto para generar resultados y curvas de aprendizaje valiosas, en especial, en zonas como el Piedemonte Llanero Colombiano, considerada una de las más difíciles de perforar a nivel mundial, primordialmente por los altos tiempos de viajes y la complejidad geológica.

Siendo consecuentes con lo anterior, en este artículo se presenta la evaluación técnica de la implementación de nuevas tecnologías que, por sus potenciales beneficios, aportan a la reducción de las principales causas de los altos tiempos de viaje, corridas de revestimientos intermedios y tiempos no productivos que han venido ocurriendo en los pozos operados por la compañía Equión Energía Limitada [3].
De esta manera se busca contribuir al mejoramiento continuo de las operaciones tratando de optimizar la perforación y el completamiento de pozos, mitigando los problemas identificados, como eventos de pega de tubería y problemas del hueco relacionados con la inestabilidad de las formaciones.

\section{GENERALIDADES DE LOS CAMPOS DEL PIEDEMONTE LLANERO COLOMBIANO}

En la cuenca de los Llanos Orientales, en la región del Piedemonte Llanero, se encuentran ubicados Campos como Pauto, Floreña, Dele y Volcanera, entre otros (Ver Figura 1). Esta corresponde a una cuenca asimétrica, localizada al este de la cordillera de los Andes y limita con Venezuela, al norte, con la cuenca del Putumayo, hacia el sur, con el sistema de fallas de Guaicáramo, al oeste, y con el sistema de fallas de Yopal-Borde Llanero, al este [6]. Para el presente artículo se estudiaron 10 pozos en los campos Pauto y Floreña.

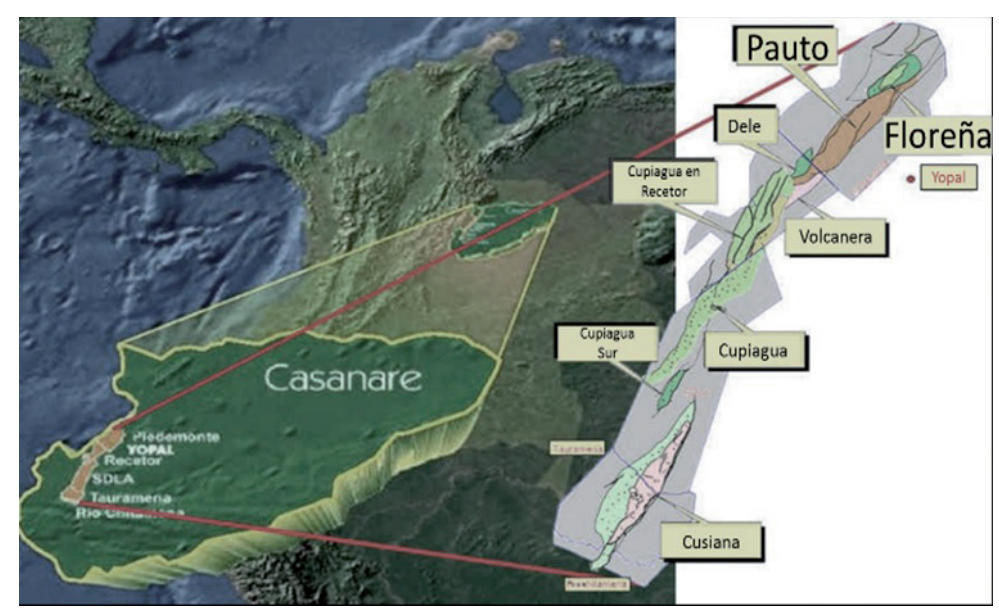

Figura 1. Campos en la región del Piedemonte Llanero Colombiano

Fuente: Tomado de CARDENAS, M. Declaración del Medio Ambiente. BP Colombia. Bogotá, 2008. Pág. 8. 
La estratigrafía en el Piedemonte Llanero se basa en principios de estratigrafía de secuencias e involucra rocas del Paleozoico, Cretáceo y Terciario con presencia de múltiples discordancias regionales [1].

Los elementos tectónicos que más afectan la cordillera oriental son el sistema de fallas de Guaicaramo, la falla de Yopal-Borde Llanero, la falla Cusiana y la falla Tamara, los cuales han generado un complejo sistema estructural, que aumenta a medida que se avanza de sur a norte. (Ver Figura 2).
La incertidumbre geológica, complejidad estructural y las características geomecánicas de la región, conllevan a un continuo monitoreo de las operaciones de perforación, durante las cuales frecuentemente se enfrentan problemas de inestabilidad de pozo y zonas de pérdidas de circulación. Estas generan restricciones en las paredes del hueco y eventos de pega de tubería que pueden representan altos tiempos no productivos.

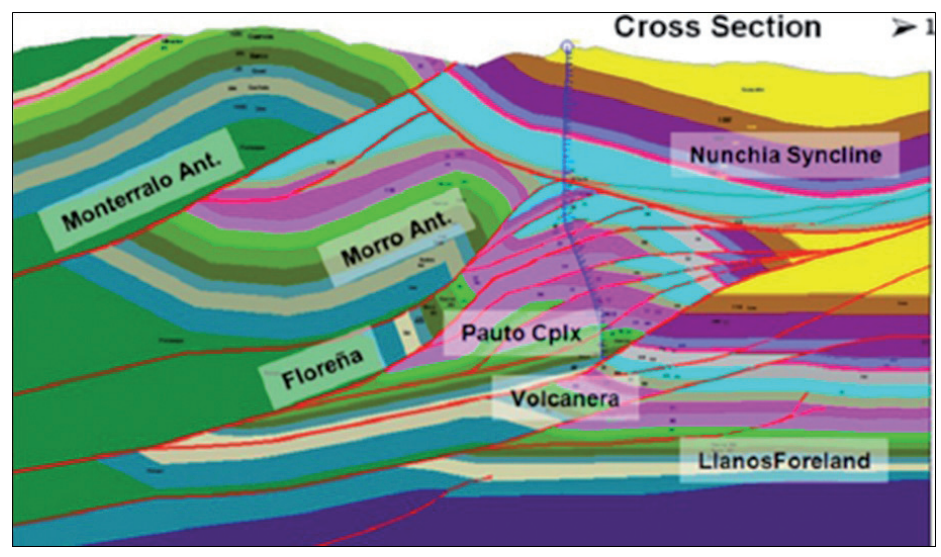

Figura 2. Sección estructural en la región del Piedemonte Llanero.

Fuente: Tomado de ALZATE, Juan Carlos. Piedemonte License. [Archivo WORD]. 2011.

\section{ANTECEDENTES OPERACIONALES}

En la región del Piedemonte Llanero se requieren altos tiempos de perforación para alcanzar los profundos yacimientos debido a las numerosas intercalaciones de arenas y lutitas, buzamientos pronunciados, gran cantidad de fallas y un ambiente tectónicamente activo. Lo anterior, a su vez, hace que se presenten numerosos problemas operacionales que se traducen en tiempos adicionales que implican mayores gastos y no hacen rentables los proyectos en la industria petrolera. Por ende, se busca constantemente reducir los principales eventos que los incrementan.

Según estudios de la compañía [2], aproximadamente el $30 \%$ del tiempo no productivo en los últimos pozos perforados, está relacionado con inestabilidad del hueco y eventos de pega de tubería. Estos resultados motivaron la implementación de nuevas tecnologías como las evaluadas en este trabajo, apuntando a la reducción de dicho porcentaje.

\section{NUEVAS TECNOLOGÍAS}

\subsection{ENSANCHADORES (UNDERREAMERS)}

Son herramientas hidráulicas concéntricas, utilizadas para ensanchar el diámetro del hueco. Tienen sistemas especiales para su activación, que les permite viajar a través del revestimiento. Su estructura de corte son unos bloques cortadores con insertos PDC, accionados por caída de bola o ball drop, que permiten operaciones de backreaming (rimar hacia arriba) y cuentan con boquillas $\mathrm{y}$ rutas de flujo en su cuerpo. Pueden ensanchar hasta un $25 \%$ del hueco piloto, según el diámetro seleccionado y se encuentran disponibles con un ciclo o múltiples ciclos de activación.

Se pueden correr para ensanchar de forma simultánea con la perforación del pozo dependiendo de la litología que se vaya a perforar (la cual debe ser uniforme) o como operación remedial para eliminar restricciones en el diámetro de un hueco ya perforado; siendo la primera 
opción la más recomendada ya que además de ser preventiva, no requiere de un BHA adicional.

Diferentes compañías de servicios tienen comercialmente disponibles ensanchadores con características distintivas.

En las operaciones de Equión Energía se decidió correr el Rhino Reamer XS (Ver Figura 3) de Smith Services (Schlumberger) y el Anderreamer de NOV; ambos con características similares y con un único ciclo de bloqueo de los bloques cortadores para ensanchar la sección de 10 5/8" a $12 "$ de diámetro.

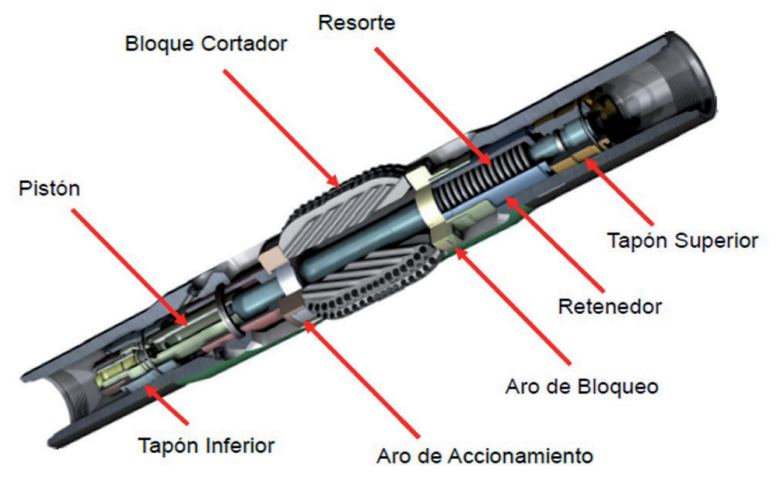

Figura 3. Configuración interna del Rhino Reamer XS

Fuente: Tomado de AVELLANEDA, Daniel. DTR Rhino XS Reamer. Schlumberger. Mayo 2013. p. 11.

\subsection{CENTRALIZADORES PROTECH CRB}

Los centralizadores estándares, en la perforación, tienen la función (entre otras) de centralizar la sarta y, en la operación de corrida de revestimiento, favorecer un espacio concéntrico para garantizar un buen trabajo de cementación. Existen diferentes tipos de centralizadores pero el implementado (Protech $C R B$ ver Figura 4) por la compañía Equion Energia Limitada, corresponde a los centralizadores rígidos. Dentro de las consideraciones de diseño del Protech CRB se contempla la mezcla de fibra de carbono con compuestos cerámicos que reducen la fricción entre las paredes del pozo y la tubería y en consecuencia, el torque y el arrastre. Es por ello que además de los beneficios que se esperan al usar cualquier tipo de centralizador [5], se evaluó la influencia de estos en los tiempos y velocidades de las corridas de revestimientos.

Los centralizadores Protech $C R B$ fueron instalados únicamente en el Pozo C8 para la corrida de casing de 11 3/4" y liner de $95 / 8$ ". En la primera, se instalaron sólo 12 centralizadores $C R B$, uno por junta, en los últimos 500 pies con el objetivo de guiar la punta del revestimiento hasta fondo. En la segunda, fueron 43 los centralizadores $C R B$ instalados en todas las juntas del liner de $95 / 8$ " con el propósito de realizar un buen trabajo de cementación y facilitar la corrida.
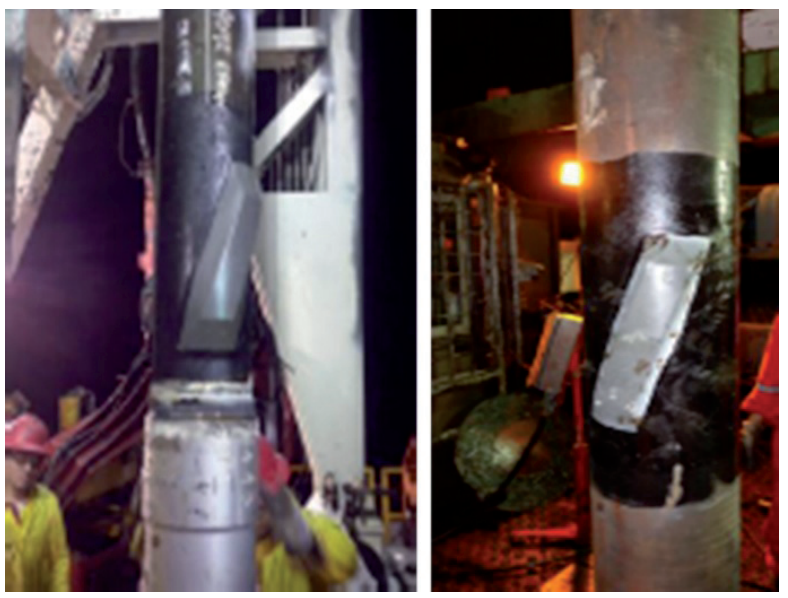

Figura 4. Centralizadores Protech CRB instalados en el Pozo C8.

Fuente: HALLIBURTON. Resultado Prueba Tecnológica Casing Resine Blade - CRB. Febrero 2014. p. 3, 5.

\section{CORRIDAS DE ENSANCHADORES Y CENTRALIZADORES}

Las secciones intermedias de los pozos $\mathrm{C} 8 \mathrm{y} \mathrm{H} 3$, fueron las seleccionadas para probar las nuevas tecnologías. En el primero, se implementaron las dos tecnologías y en el segundo, únicamente el ensanchador Rhino Reamer de Smith.

En la sección de 14 3/4", la más larga en la mayoría de proyectos, se instalaron centralizadores PROTECH $C R B$ en $500 \mathrm{ft}$ del casing de $113 / 4$ " para que sirvieran de guía a la cola del revestimiento en el Pozo C8. Cabe anotar que en los últimos seis años no se han utilizado centralizadores, ni rígidos ni ajustables, debido a que al necesitar en la mayoría de casos trabajar el revestimiento para superar condiciones de inestabilidad, características de la zona, se estaban provocando pérdidas de estos dispositivos en fondo. El diseño innovador desarrollado por la compañía Halliburton en estos centralizadores, parecía reducir esas limitaciones, y por tal razón se volvió a considerar su implementación.

En la siguiente sección, además de usar centralizadores en todas las juntas del liner de $95 / 8$ ", se ensanchó el hueco de $105 / 8$ " hasta 12 ", al mismo tiempo que se 
perforaba en el Pozo C8. En cambio, en el Pozo H3 se realizó el ensanchamiento luego de haber perforado el intervalo. La primera operación de ensanchamiento fue una acción preventiva ante el alto riesgo de puntos apretados y manifestaciones de inestabilidad del pozo, contrario al caso del $\mathrm{H} 3$, donde se realizó para mejorar las condiciones de un hueco ya deteriorado, al punto de impedir llegar a fondo con cuatro ensamblajes.

\section{EVALUACIÓN Y ANÁLISIS DE RESULTADOS}

\subsection{METODOLOGÍA UTILIZADA}

Se definió una secuencia estructurada de actividades para cumplir los objetivos trazados en el estudio.

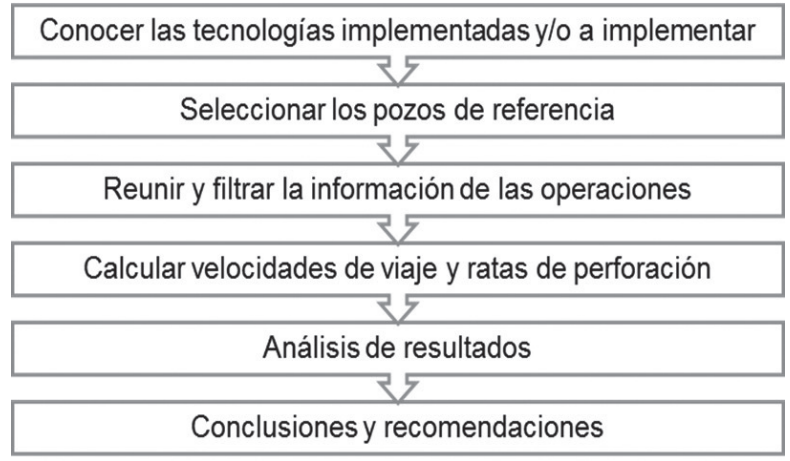

Figura 5. Metodología para evaluación y análisis

Luego de conocer los aportes que se esperaban con las nuevas herramientas y seleccionar los mejores pozos de correlación para comparar resultados, los reportes diarios de perforación, reportes finales, guías de perforación y estados mecánicos fueron la base central de la información estudiada para evaluar el impacto de las nuevas tecnologías y su posible recomendación para futuros proyectos. Dado lo extensos que pueden resultar, todo se enfocó exclusivamente en las actividades y eventos ligados con la estabilidad del hueco durante los viajes y corridas de revestimiento. Se incluyeron los tiempos no productivos por baja calidad del pozo durante estas actividades, debido a que con las nuevas tecnologías también se apuntaba a su reducción.

Como se tenía definido calcular velocidades de viaje de tubería tanto sacando $(\mathrm{POOH})$ como entrando (RIH) y velocidades de corrida de revestimientos netas, se filtró la información para no tener en cuenta tiempos por toma de parámetros y registros, actividades HSE, reparaciones de equipos, viajes para curar pérdidas, tiempos en hueco revestido ni tiempos de circulación, exceptuando los necesarios para enfrentar empaquetamientos y restricciones al viajar por las paredes del pozo. Resultando además este cálculo efectivo para normalizar los tiempos con las distancias recorridas.

Los viajes de acondicionamiento se incluyeron en los análisis de corridas de revestimiento y no en tiempos de viaje.

Posteriormente se compararon resultados con los presentados en los pozos con condiciones similares a las del pozo piloto, como perfil direccional y formaciones geológicas. Este último factor es crítico en secciones agresivas como la de $105 / 8$ " donde se corre revestimiento con solo 1" de anular. Las formaciones C7 y C8, inestables, hinchables y muchas veces intercaladas, son las que presentan condiciones a mejorar con las nuevas tecnologías.

Para facilitar el análisis de los datos obtenidos, se graficaron barras rellenas con los espesores de las formaciones de cada sección de pozo (Tabla 1), a escala, para correlacionar velocidades de viaje con litologías, esperando mayores tiempos de ejecución en los pozos con las formaciones citadas previamente.

Tabla 1. Colores que representan cada Formación

\begin{tabular}{|c|c|c|c|c|c|c|}
\hline \multicolumn{4}{|c|}{ Formaciones Carboneras } & \multirow{2}{*}{ Mirador } & \multirow{2}{*}{$\begin{array}{c}\text { Los } \\
\text { Cuervos }\end{array}$} & \multirow{2}{*}{ Barco } \\
\hline C5 & C6 & C7 & C8 & & & \\
\hline
\end{tabular}

\subsection{VELOCIDADES DE VIAJE PROMEDIO EN HUECO ABIERTO}

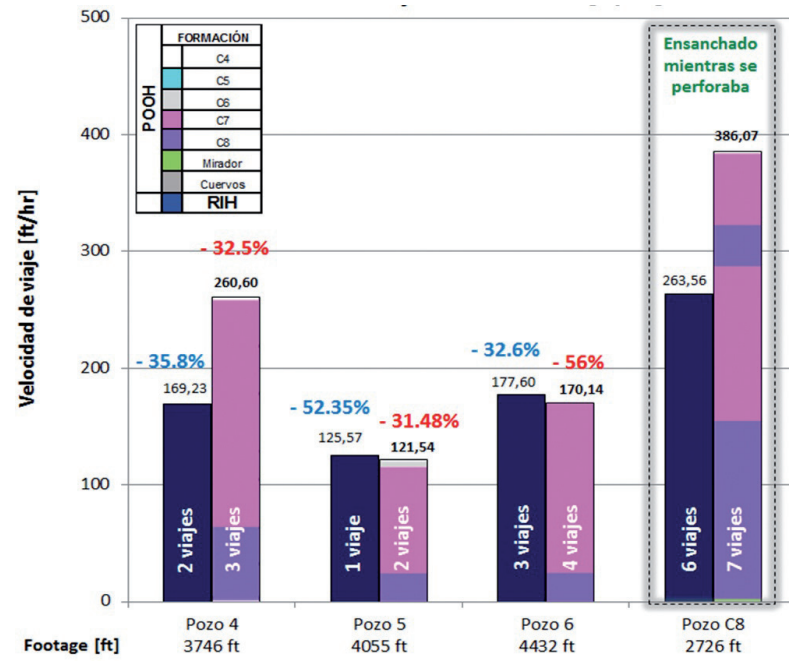

Figura 6. Velocidades de viaje RIH \& POOH en 10 5/8" 
Para comparar los viajes de tubería son relevantes sólo los datos de la sección de $105 / 8$ " donde se usaron los ensanchadores. En la Figura 6 están las velocidades de viaje netas calculadas para tres pozos de correlación junto a las del pozo en evaluación, el C8; siendo evidentemente superiores los resultados de este al viajar bajando y sacando el ensamblaje. Para cuantificar las mejoras alcanzadas, se calcularon las variaciones porcentuales con la ecuación (1):

$$
\text { Variación }(\%)=\frac{\text { Velocidad pozo } X * 100 \%}{\text { Velocidad pozo C8 }}-100
$$

Obteniendo velocidades superiores al 32,5\% en viajes POOH y al $32,6 \%$ en viajes RIH.

Adicionalmente, se estudiaron los perfiles direccionales de cada pozo para verificar si las diferencias entre las operaciones se pudieron generar por secciones más complejas pero no se encontró relación alguna, diferente a la reducción de eventos de empaquetamiento y puntos trabajados que obligaban a trabajar la sarta. Incluso la sección del Pozo C8, aunque tangente, se mantuvo con mayores inclinaciones $\left(23^{\circ}-25^{\circ}\right.$ de inclinación).

\subsubsection{Distribución de LOS TIEMPOS DE VIAJE.} Como se dijo previamente, en el hueco ensanchado de $105 / 8$ " a 12", el incremento de las velocidades de viaje estuvo relacionado directamente con la reducción de restricciones en las paredes del pozo. Esto se debe a que en los viajes libres (con bombas y/o en elevadores) no hay necesidad de rotar la sarta y por tanto, se realiza la operación en menos tiempo.
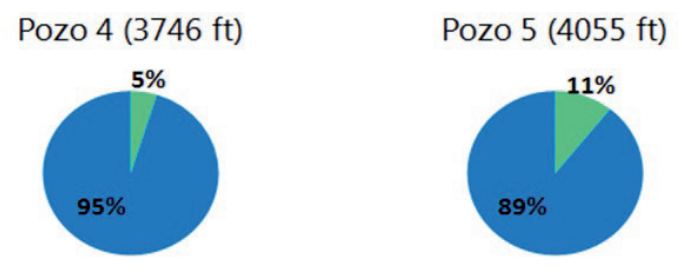

Pozo 6 (4432 ft)

Pozo C8 (2726 ft)
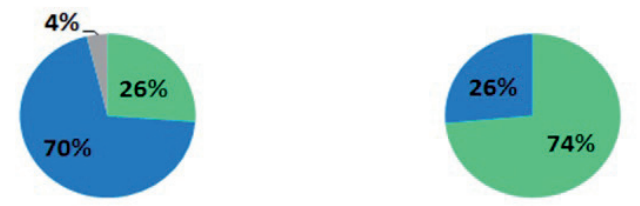

Washing/en Elevadores a Rmg/Brmg (hrs) =NPT DPRB (hrs)

* NPT DPRB $=$ NPT por problemas en fondo de hueco

Figura 7. Distribución de tiempos RIH sección de 10 5/8"

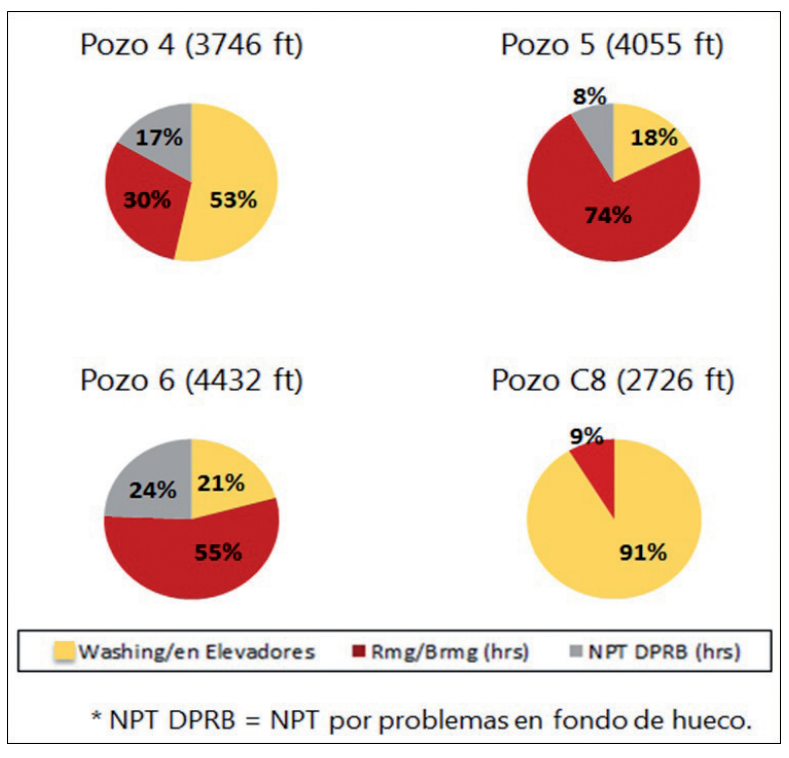

Figura 8. Distribución de tiempos POOH sección 10 5/8"

\subsection{COMPORTAMIENTO DE LA ROP}

Se comparó la ROP del Pozo C8 con los demás pozos de correlación (tres de ellos aparecen en la Figura 9).

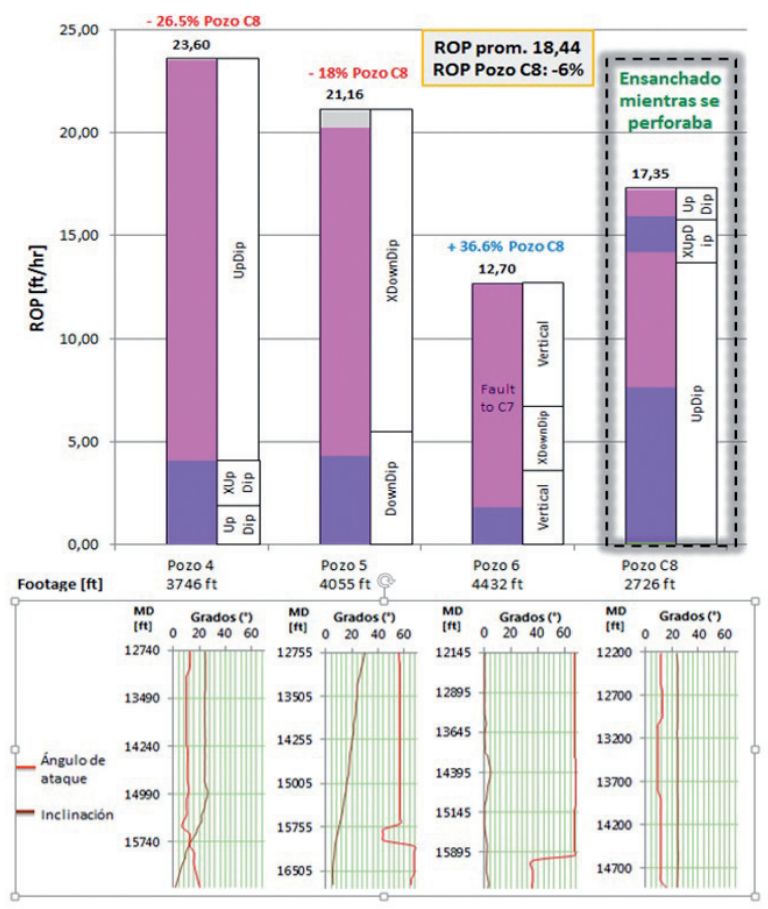

Figura 9. Impacto en ROP al usar ensanchador

Al tener una estructura de corte adicional a la broca (los bloques cortadores del ensanchador), se esperaba un impacto negativo en la tasa de penetración (ROP); 
importante de cuantificar, de tal forma que al evaluar los beneficios alcanzados, se estableciera la viabilidad de la operación de ensanchamiento durante la perforación de la sección de 10 5/8" por formaciones carboneras. En la parte superior de las barras de la Figura 9, se muestra el impacto de cada pozo y una ROP promedio que se obtuvo considerando dos pozos adicionales, cuyas formaciones geológicas eran similares en esta sección. Finalmente se obtuvo una reducción promedio del 6\% en la tasa de perforación. La ROP efectiva en el Pozo C8 de 17,35 ft/hr fue mayor que la tasa simulada y esperada por la compañía de servicios de 14,7ft/hr. Más importante aún, la ROP pudo ser significativamente mayor, de no haber corrido el ensanchador con parámetros controlados por ser la primera vez que se implementaba en las operaciones de Equión.

No se encontró relación alguna entre el relative bearing (ángulo entre la trayectoria y la dirección de buzamiento, comúnmente como componente del máximo esfuerzo horizontal) y la ROP donde se podrían esperar menores tasas de penetración al ir cross-dip (perpendicular a la capa). Los ángulos de ataque si influyeron en el desempeño de la ROP de cada pozo ya que al perforar con la broca perpendicular al plano de estratificación (tendencia natural) se favorece la estabilidad mecánica y con ella el avance en profundidad. Es decir, a medida que se aleja de la tendencia natural, aumentando el ángulo, se incrementa el riesgo de presentar eventos de inestabilidad en el pozo durante la perforación, asociados a fuerzas compresivas y distorsionales. En general, los de ángulos de ataque más favorables $\left(<20^{\circ}\right)$ permiten perforar con una mayor ROP [4].

\subsection{CASO POZO H3: ENSANCHAMIENTO REMEDIAL DE SECCIÓN PERFORADA}

La decisión de correr un ensanchador en este pozo no buscaba prevenir problemas de estabilidad que afectaran la calidad del hueco como en el Pozo C8, sino reducir y remediar los que ya se estaban presentando al intentar perforar hasta la profundidad total (TD) de la sección. El deterioro de las condiciones del hueco era tan grave, que había sido imposible llegar a fondo con cuatro BHA. El undécimo viaje, que enfrentó tres empaquetamientos, fue determinante para decidir correr el Rhino Reamer XS 10000 .

Aunque se alcanzó a perforar hasta $14.139 \mathrm{ft}$ de la profundidad medida (MD), las restricciones en el intervalo posteriormente ensanchado impedían volver a fondo. En total fueron $1.110 \mathrm{ft}$ ensanchados hasta 12" de diámetro (Ver Figura 10). Los viajes realizados en la sección se representan en la Figura 11 (calculados en paradas/hora).

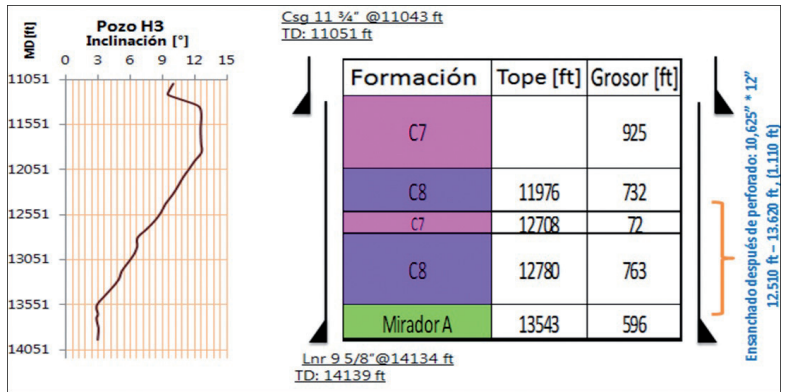

Figura 10. Sección de hueco de 10 5/8" del Pozo H3

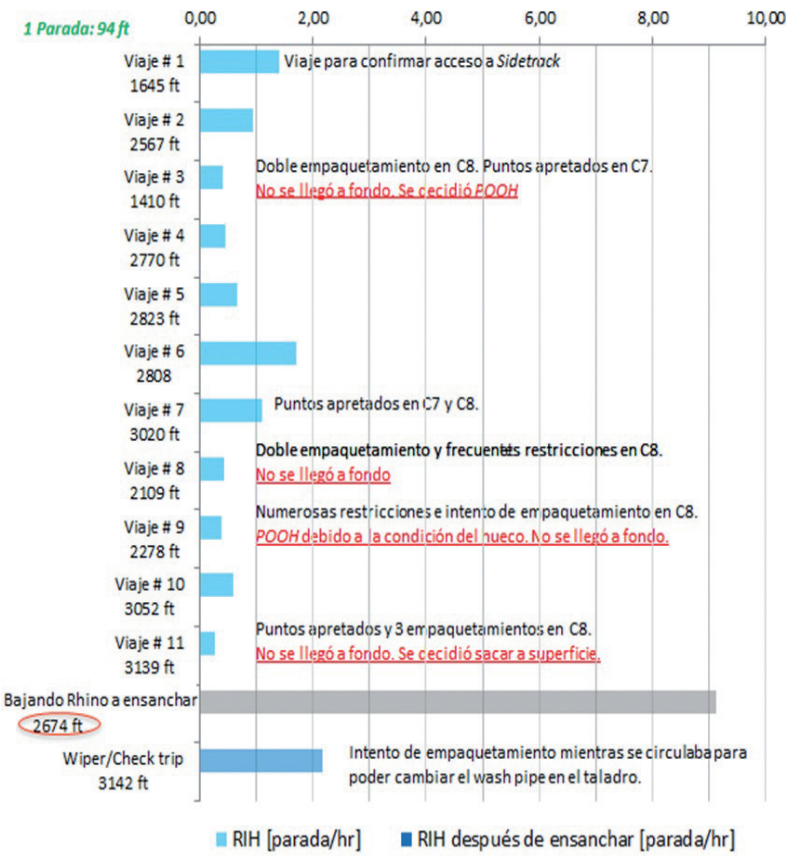

Figura 11. Viajes RIH sección de 10 5/8” Pozo H3

La velocidad de 9,11 paradas/hora no se debe a los beneficios del ensanchamiento. Si se observa en el eje vertical, a comparación de los dos viajes previos, solo recorrió $2.674 \mathrm{ft}$ en hueco abierto (hasta $12.449 \mathrm{ft} \mathrm{MD}$ ), es decir, se detuvo antes de pasar por el intervalo donde más ocurrían atascamientos de la tubería y que justamente iba a ser ensanchado luego desde $12.510 \mathrm{ft}$ MD.

Durante todos los viajes RIH se presentaron numerosos puntos apretados y en total 10 eventos de empaquetamientos de la sarta; mientras que sacando tubería las restricciones fueron inferiores y se presentaron solo dos empaquetamientos, como se diferencia fácilmente en las velocidades graficadas para ambos casos. 


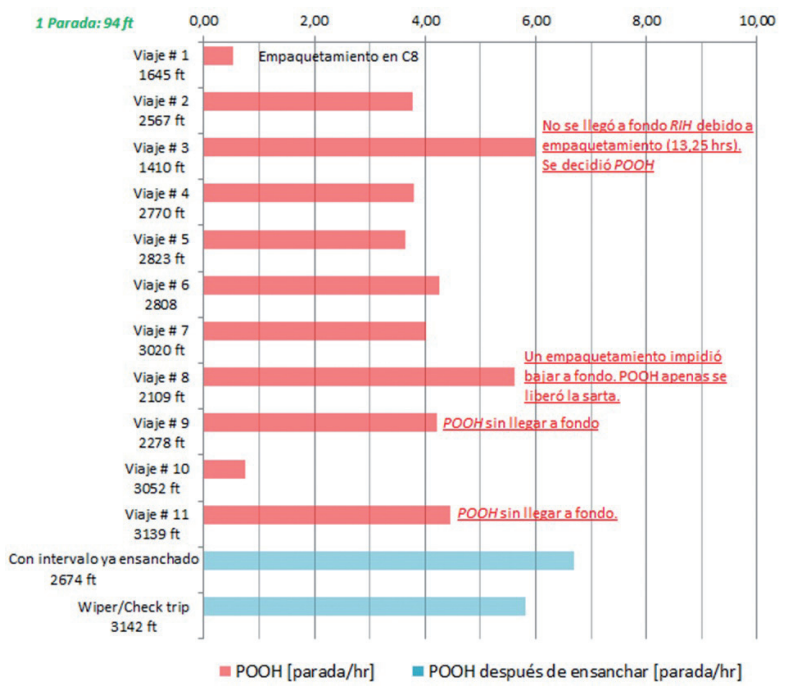

Figura 12. Viajes POOH sección de 10 5/8" Pozo H3

Si bien se observan aumentos en las velocidades de entrada y salida de tubería, lo principal era mejorar la calidad del hueco, evidentemente deteriorado, con el ensanchamiento del intervalo definido para lograr regresar a fondo después de cuatro fracasos, y culminar la sección para correr el revestimiento de $95 / 8$ ".

A pesar de los buenos indicios experimentados en los viajes realizados con el intervalo ensanchado, se temía encontrar nuevamente dificultades en el hueco. Por lo tanto, lo más importante no era el tiempo que se tardará la corrida del revestimiento sino sentarlo en fondo, lo cual se logró dejando el zapato a solo $5 \mathrm{ft}$ de TD. Sólo se presentaron dos leves restricciones a $13.278 \mathrm{ft}$ y 13.288 $\mathrm{ft}$ de MD, superadas con un poco de rotación del liner. Además, es relevante resaltar que no hubo inconvenientes con las variaciones de diámetro del hueco ni pérdidas de circulación durante la ejecución de la operación.

Considerando todo lo anterior, se puede afirmar que la operación de ensanchamiento como acción remedial, fue exitosa.

\subsection{CORRIDAS DE REVESTIMIENTO}

5.5.1 CASING DE 11 3/4" EN HUECO ABIERTO. Para realizar la comparación entre las corridas de revestimientos se calcularon dos velocidades (vel.) de corrida (en ft/ hr): una considerando horas de corrida en hueco abierto con horas completas del viaje de acondicionamiento o wiper trip (ecuación 2) y otra sólo con tiempos de corrida (ecuación 3).

$$
\text { Vel. con Wiper Trip }=\frac{\text { Longitud hueco abierto }}{\text { tiempo de: } \text { corrida }+ \text { wiper trip }}
$$

Vel. sin Wiper Trip $=\frac{\text { Longitud hueco abierto }}{\text { tiempo de corrida en hueco abierto }}$ (3)

En todos los pozos se hizo un viaje de acondicionamiento antes de bajar a revestir el hueco de $143 / 4$ ". Las mejores velocidades se dieron en los Pozos 4, 7x, 9 y C8 (Ver Figura 13). Resaltando el desempeño logrado en el Pozo 9 cuya sección igual que la mayoría de la del pozo ensanchado, atravesaba las complejas condiciones de formaciones $\mathrm{C} 7 \mathrm{y} \mathrm{C} 8$.

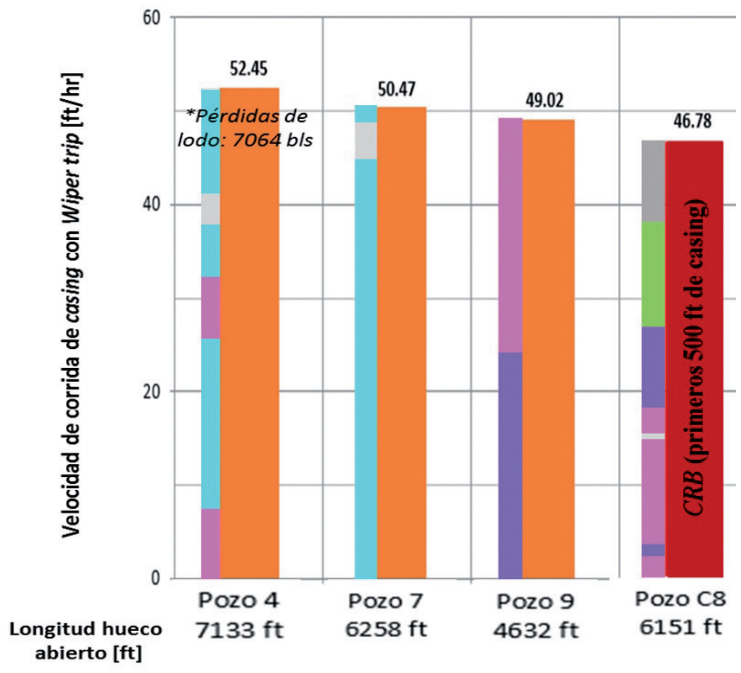

Figura 13. Velocidades de corrida de casing de 11 3/4" en hueco abierto incluyendo wiper trip.

En la Figura 14 se separaron los tiempos para acondicionar el hueco de los de velocidad de corrida. Aunque el wiper trip es poco cuestionado en esta sección tan larga se observa que gastar más horas acondicionando el hueco no se traduce directamente en corridas más veloces a fondo.

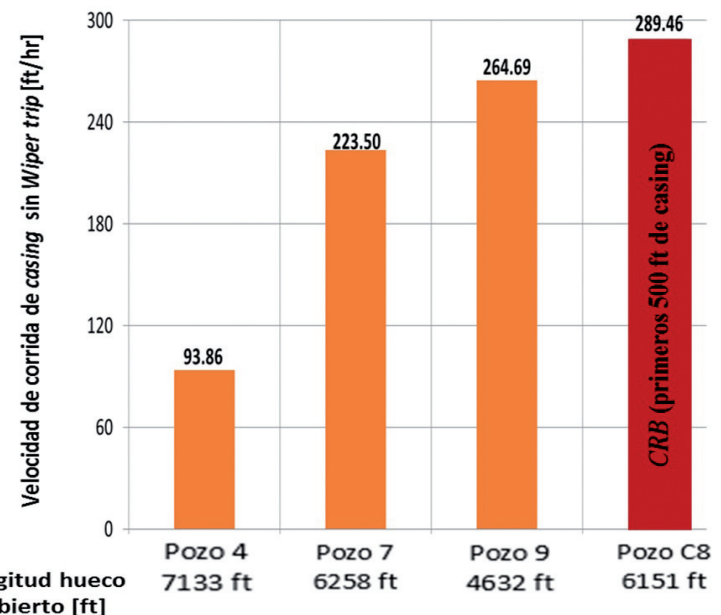

Figura 14. Velocidades de corrida de casing de 11 3/4" en hueco abierto sin wiper trip. 
La mayor velocidad netamente de corrida del casing de 11 3/4 en hueco abierto fue la del Pozo C8 a 289,46 ft/ $\mathrm{hr}$, lograda por las excelentes condiciones del hueco y, posiblemente, los nuevos centralizadores reductores de fricción.

Otro factor influyente en estos tiempos, es la trayectoria del pozo. Por eso se analizaron los perfiles direccionales de los 4 mejores pozos (4, 7x, 9 y C8). Los cambios de ángulo pueden aumentar los puntos de contacto entre el pozo y las juntas del revestimiento y llegar a restringir el avance a fondo. En los tres pozos con mayor velocidad en hueco abierto $(7 \mathrm{x}, 9$ y C8) se construyó ángulo, pero considerando conjuntamente las formaciones, los resultados del Pozo C8 siguen siendo los mejores.

\subsubsection{Revestimiento de $\mathbf{9}$ 5/8" en hueco} ABIERTO. En la corrida de este revestimiento, frecuentemente se ha hecho necesario trabajarlo para llevarlo a fondo, comprometiendo la integridad del mismo. Debido a esto, lo que se pretende es reducir las horas de trabajo (torque y rotación), que están

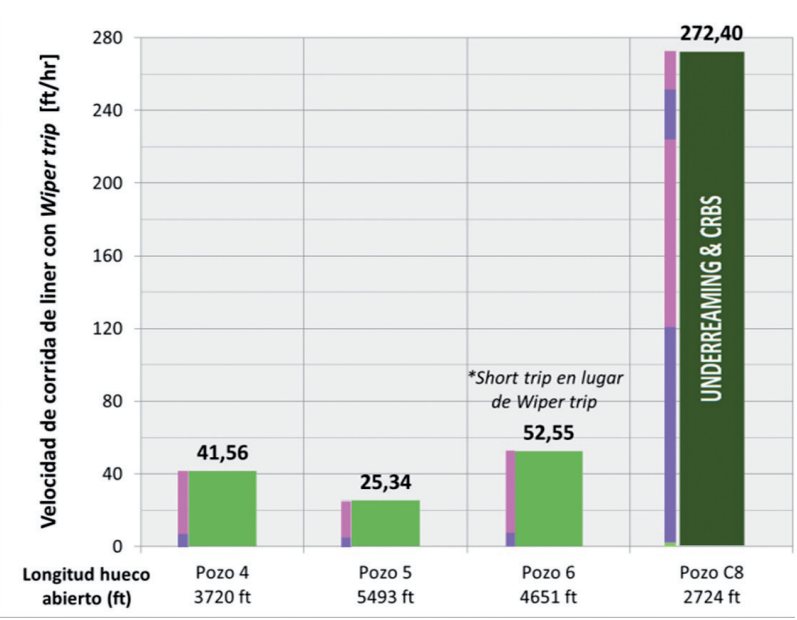

Figura 15. Velocidades de corrida de revestimientos de 9 5/8" en hueco abierto considerando tiempo de wiper trip

\subsection{ESTUDIO DE TIEMPOS PLANOS O FLAT TIMES}

Un tiempo plano es aquel durante el cual no se obtiene progreso en la profundidad del pozo para alcanzar la profundidad final del mismo. Para este estudio, sólo se consideraron los tiempos planos que transcurrieron entre el último pie del hueco de $105 / 8$ " y el primer pie perforado de la sección de $81 / 2$ ". Los tiempos planos a los que se hace referencia son: viaje $\mathrm{POOH}$, registros, problemas en fondo de hueco y ensanchamiento, representando más tiempo del planeado. Además, por las características de la sección de $105 / 8$ ", usar centralizadores Protech $C R B$ que no restringen el flujo del lodo como los centralizadores rígidos o ajustables, pueden resultar factibles ante estrechas ventanas operativas de ECD (densidad equivalente de circulación) que pueden presentarse.

El pozo C8 fue en el único que se corrió el revestimiento sin utilizar previamente un ensamblaje de acondicionamiento. El Pozo M4, en la Figura 16, muestra la mejor velocidad de corrida; sin embargo, al considerar las horas de acondicionamiento del hueco (wiper trip), se aprecia como su velocidad disminuye considerablemente (Figura 15). En ese sentido, al compararlo con el Pozo C8, aparentemente los viajes de acondicionamiento en esta sección no son indispensables para garantizar una corrida exitosa y veloz. El tiempo de preparación del hueco no garantiza una reducción de los mismos en las operaciones posteriores y en cambio pueden producir mayor grado de deterioro.

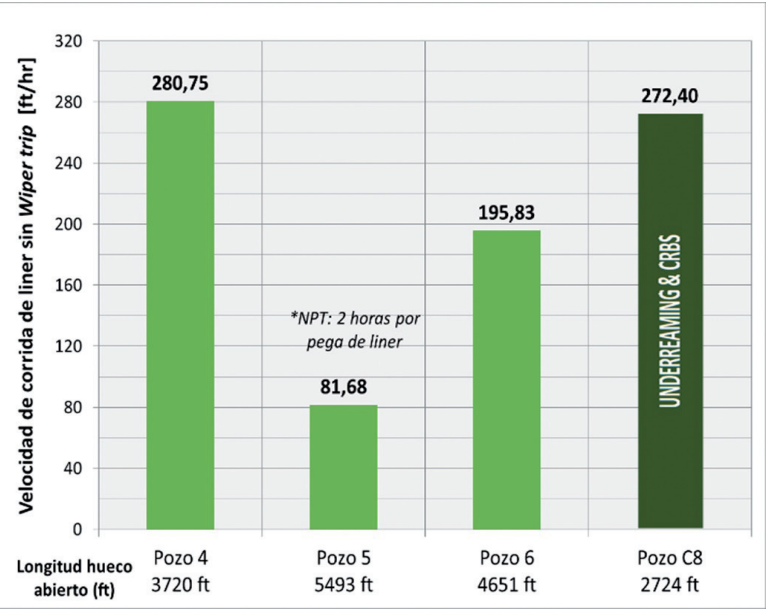

Figura 16. Velocidades de corrida de revestimientos de 9 $5 / 8$ " en hueco abierto sin considerar wiper trip.

acondicionando el hueco para correr liner/casing (wiper trip), corrida de liner/casing, cementación, prueba de BOP, acondicionando el zapato, NPT y otras actividades en las que no se perfora un solo pie de formación.

El análisis de los flat times se realizó para complementar la evaluación presentada anteriormente de las tecnologías, buscando al igual que en cualquier otra actividad de la fase de perforación, el menor tiempo posible como lo ideal. 


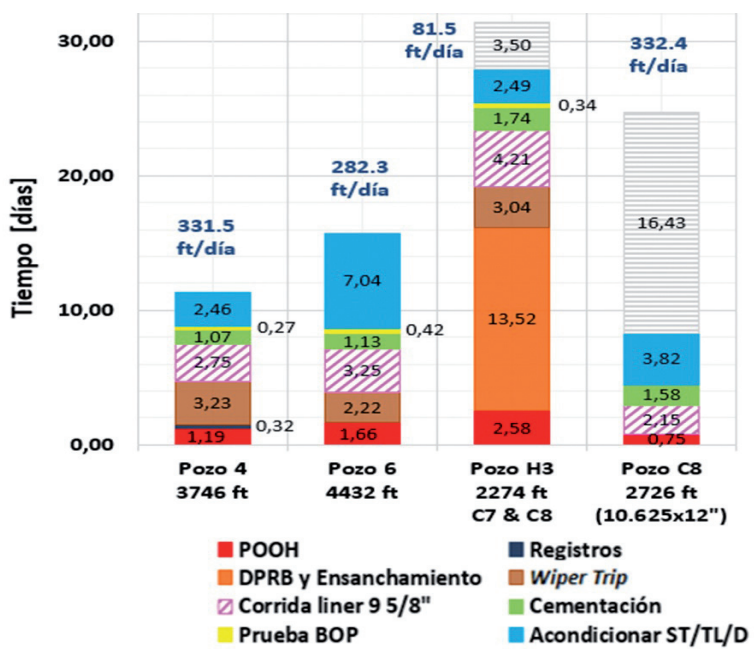

Figura 17. Tiempos planos previos a la corrida de revestimiento de $95 / 8$ "

Los Pozos $\mathrm{H} 3$ y C8, los cuales implementaron la tecnología de ensanchador, no son los de menores flat times (Ver Figura 17). Sin embargo, esto se debe, en el caso del Pozo H3, al tiempo perdido por los problemas en fondo del hueco que conllevaron a la ampliación del mismo como trabajo remedial y, en el caso del Pozo C8, a tiempo curando perdidas y pescando una pieza de la herramienta de corrida del liner.

En ese sentido y con el fin de analizar mejor los resultados de las nuevas tecnologías, se separaron los tiempos planos de los tiempos no atribuibles a las condiciones del hueco (tiempos no productivos, NPT). Dentro de los NPT considerados, es relevante mencionar los asociados a la integridad de los topes de los liner por alto trabajo mientras se llevan a fondo. La separación consistió en normalizar el tiempo en días de los flat times con la longitud de la sección. Los valores normalizados de los pozos se muestran en la parte superior de las columnas. Se puede apreciar, para el caso del Pozo C8, un valor normalizado más representativo de $332.4 \mathrm{ft} /$ día.

\section{IMPACTO EN COSTOS}

Se realizó un análisis del impacto en los costos como parte final de la evaluación técnica de las nuevas tecnologías implementadas para reducir tiempos de viajes y tiempos de corridas de revestimientos, con el propósito de cuantificar el tiempo y, por ende, dinero ahorrado por la compañía Equión Energía Limitada en sus operaciones en pozos del Piedemonte Llanero Colombiano.

Para ello se diseñó un flujo de caja que permitiera cuantificar el ahorro alcanzado con los resultados obtenidos de la operación de ensanchamiento, los centralizadores Protech CRB y, adicionalmente, los tiempos planos.

\subsection{ENSANCHADORES (UNDERREAMERS)}

Con base en el desempeño de cada operación evaluada en hueco abierto, se convirtió la relación porcentual obtenida en los resultados de velocidades de viaje, ROP y wiper trip en su equivalente monetario y de esta manera, se estudió si el tiempo ahorrado en la ejecución de las operaciones del Pozo C8, justificó la inversión en los ensanchadores.

\begin{tabular}{|c|c|c|}
\hline \multicolumn{3}{|l|}{ Horas ahorradas: } \\
\hline VIAJANDO $\mathrm{POOH}=$ & 8.5 & \\
\hline VIAJANDO RIH = & 8.3 & \\
\hline PERFORANDO (ROP) = & -9.3 & \\
\hline SIN WIPER TRIP = & 53.5 & \\
\hline Total horas ahorradas $=$ & $8.5+8.3-9.3$ & $+53.5=61$ \\
\hline Costo diario de perforación $=$ & $\$ 170,000.00$ & U\$D/dia \\
\hline Dinero ahorrado $=$ & $\frac{\$ 170,000 * 61}{24}$ & $-=\$ 432,083.33$ \\
\hline Costo por pie ensanchado $=$ & $\$ 53.00$ & USD/ft \\
\hline Inversión de ensanchamiento $=$ & $\$ 53^{*} 2612$ & $=\$ 138,436.00$ \\
\hline Total ahorrado $=$ & $\$ 293,647.33$ & \\
\hline
\end{tabular}

Figura 18. Cálculos para determinar el ahorro total que representó la operación de ensanchamiento

Además del ahorro de \$293,647.33 dólares que se obtuvo con la operación de ensanchamiento, es de vital importancia resaltar que el uso de los ensanchadores minimizan los riesgos de pegas de tubería que podrían desencadenar un sidetrack en esta sección e impactar en costos por más de 2 millones de dólares $(\$ 2,000,000.00)$, tal y como sucedió en 3 casos presentados dentro de los diez pozos en estudio. De igual forma, el total ahorrado sería mayor si se perforará sin parámetros controlados, como ocurrió en esta ocasión, por ser la primera vez que se corría la herramienta y además, se optimizará la perforación con cortadores de $16 \mathrm{~mm}$ en lugar de $13 \mathrm{~mm}$.

\subsection{CENTRALIZADORES PROTECH CRB}

Los centralizadores protech $C R B$, en las corridas de casing de 11 3/4" y liner de $95 / 8$ " del pozo C8, significaron para la empresa Equión Energía limitada una inversión total de \$306.696 dólares. 
Los centralizadores dependiendo de la sección donde fueron instalados, cumplieron con su función. Por ejemplo, en la sección de 14 3/4" para la corrida de casing de $113 / 4$ " el principal objetivo era guiar la punta del revestimiento a fondo, superando escalonamientos de formaciones con washouts; mientras que, en la sección de 10 5/8" para la corrida de liner de $95 / 8$ " el principal objetivo era cumplir con un buen trabajo de cementación.

Si bien solo se presenta el impacto en costos de los centralizadores como una inversión, es de gran importancia señalar que el uso de los mismos evita tiempo trabajando el revestimiento para llevarlo a fondo e inversiones adicionales en trabajos remediales de cementación como consecuencia de la pérdida de integridad del liner en conexiones o topes del mismo, además de reducir el riesgo de necesitar correr scab liner.

\subsection{FLAT TIMES}

Como última propuesta para analizar el impacto en costos de las nuevas tecnologías que fueron objeto de estudio en esta tesis de grado, se presenta el ahorro obtenido al lograr la reducción de los tiempos planos del Pozo C8 en la sección de 10 5/8".

Para el cálculo del dinero total ahorrado, en un principio se normalizaron las velocidades de flat times (en $\mathrm{ft} /$ días) de los Pozos 4, 5, 6, 10, H3 y 7x con la longitud de la sección perforada del Pozo C8. Luego, se promediaron los días obtenidos en la normalización y se restaron con el tiempo plano (en días) del Pozo C8 (no se incluyen los días perdidos por fallas mecánicas ni perdidas de circulación). La diferencia anterior corresponde al tiempo ahorrado como resultado de la implementación de las nuevas tecnologías.

Por último, se multiplicaron los días obtenidos como impacto en el tiempo por el promedio entre el costo diario de perforación y corrida de revestimiento.

La implementación de las nuevas tecnologías permitió superar el millón de dólares en dinero ahorrado. Al restarle las inversiones del ensanchamiento y de los centralizadores instalados para la corrida del liner de 9 5/8", el total ahorrado fue de $\$ 907,627.27$ (ver Figura 19).

En conclusión, por lo demostrado en el Capítulo 6 con el análisis de resultados y en este con la evaluación del impacto en costos, queda comprobada la rentabilidad de la operación de ensanchamiento y de los centralizadores Protech $C R B$, tanto en la parte práctica como en la económica.

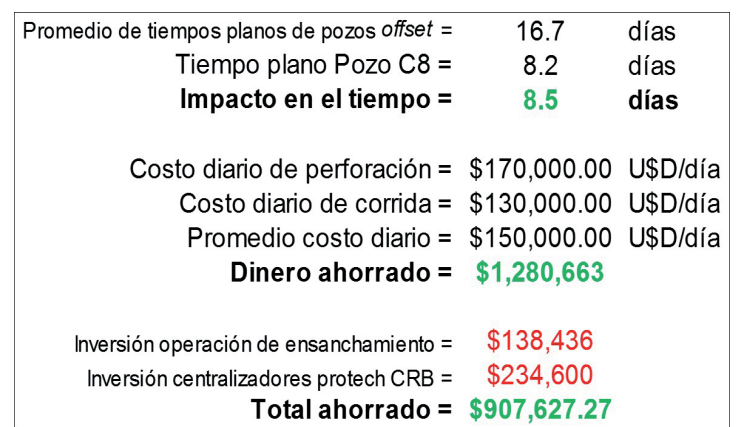

Figura 19. Impacto en costos de los tiempos planos del Pozo C8 en la sección de $105 / 8$

\section{CONCLUSIONES}

En las secciones intermedias estudiadas (143/4" y 10 5/8") el promedio de velocidades sacando tubería del hueco $(\mathrm{POOH})$ fue significativamente mayor al promedio de velocidades entrando tubería al hueco $(\mathrm{RIH})$ en un $86 \%$ de los casos.

Las velocidades promedio viajando a través de las Formaciones C7 y C8 del Pozo C8, en la sección de 10 $5 / 8$ ", aumentaron entre un $33-52 \%$ entrando al hueco (RIH) y entre un 32-56\% sacando del hueco (POOH) al reducir puntos apretados y eventos de pega de tubería con la operación de ensanchamiento.

Durante la operación de ensanchamiento del Pozo C8, en la sección de $105 / 8$ ", perforando las formaciones de C7 y C8 se observó un impacto negativo en la rata de penetración (ROP) del 6\% comparada con los cuatro pozos de correlación (offset).

A pesar de los problemas de estabilidad de hueco que se presentaron durante la perforación de la sección de 10 5/8" del Pozo H3, la exitosa operación remedial de ensanchamiento permitió llegar a fondo luego de cuatro corridas de BHA deficientes y correr liner de $95 / 8$ " sin restricciones hasta fondo.

La operación de ensanchar mientras se perfora puede evitar la necesidad de realizar wiper trip, logrando reducir el tiempo de exposición del hueco antes de correr el revestimiento y garantizar una mejor calidad del pozo.

Basados en el análisis de tiempos e impacto en costos se puede afirmar que la implementación de la tecnología de los centralizadores $C R B$ cumplió con su objetivo en la sección de 14 3/4" de ayudar a llevar el casing de 11 3/4" a fondo, minimizando el tiempo de corrida y trabajo del mismo, y en la sección de $105 / 8$ " mejorando el trabajo de cementación del liner de 9 5/8". 
Los buenos resultados obtenidos en la corrida de revestimiento de 9 5/8" en el Pozo C8 se deben a la implementación, en conjunto, de las nuevas tecnologías ensanchador y centralizadores $C R B$; comprobando que al minimizar el tiempo de exposición del hueco, este muestra unas mejores condiciones para viaje y corrida de liner.

\section{REFERENCIAS}

1. ALZATE, Juan Carlos. Piedemonte License. [Archivo WORD]. 2011. 8 p. Tomado de: GUTIÉRREZ, Stephanie. Selección del sistema de perforación direccional más adecuado para las operaciones de perforación en el Piedemonte Llanero mediante una evaluación de desempeño. Trabajo de grado para optar al título de Ingeniero de Petróleos. Bogotá D.C.: Universidad de América. 2012. p. 32.

2. BAKER HUGHES. Well of the Future. Connexus, vol. 4 , no. 1, p. $4-11,2013$.

3. LÓPEZ, Sergio y PALACIOS, Iván. Evaluación técnica de la implementación de nuevas tecnologías para reducir tiempos de viajes y tiempos de corridas de revestimientos durante la perforación de pozos en campos petrolíferos del Piedemonte Llanero
Colombiano. Trabajo de grado para optar al título de Ingeniero de Petróleos. Bucaramanga, Colombia: Universidad Industrial de Santander. Facultad de Ingenierías Físico-químicas. Programa de Ingeniería de Petróleos, 2014. 188 p.

4. LOPEZ, Cesar y ROA, Aura. Efecto del ángulo de ataque en la perforación de pozos en el piedemonte llanero. En: Simposio de Geomecánica (5: 1, marzo, 2013: Medellín, Colombia). Medellín: 2013. 21 p.

5. SANCHEZ, Renato and ADAMS, Whitney. Casing Centralization in Horizontal and Extended Reach Wells [online]. Society of Petroleum Engineers, 2012 [cited june 2014]. Available from Internet: <URL: https://www.onepetro.org/conference-paper/ SPE-150317-MS>.

6. VIRGILIO y NARANJO, et al. 2004. Tomado de: ROA, Aura María. Análisis, causas y caracterización de cavings aplicado a la estabilidad de pozos: dos casos en el Piedemonte Llanero. Trabajo de grado para optar al título de Geólogo. Bucaramanga: Universidad Industrial de Santander. Facultad de Ingenierías Físico-químicas. Programa de Geología, 2006. p. 17-31.

Recepción: 1 de Julio de 2015

Aceptación: 23 de Septiembre de 2015 